\title{
Persistent left sided fifth aortic arch in a neonate
}

\author{
ALBERTO CABRERA, JOSE GALDEANO, IÑAKI LEKUONA \\ From the Department of Paediatric Cardiology, Hospital Infantil Cruces, Bilbao, Spain
}

SUMmaRY A persistent left sided fifth aortic arch with coarctation of the aorta and persistence of the ductus arteriosus was recognised and treated surgically in a newborn infant. The fifth arch was used to repair the coarctation, and five years later the child had normal peripheral pulses and no residual murmurs.

Persistence of the fifth aortic arch is a rare congenital defect. It has been left sided and associated with tricuspid atresia and cor triatriatum, 12 coarctation of the aorta, ${ }^{3}$ persistence of the ductus arteriosus, ${ }^{4} 5$ and with pulmonary atresia and ventricular septal defect. ${ }^{67}$ Necropsy findings have been reported in four of these cases.

\section{Case report}

A male infant (birth weight $3 \mathrm{~kg}$ ) born after a full term pregnancy and normal delivery showed respiratory distress and slight cyanosis 24 hours later. The liver was palpable $3 \mathrm{~cm}$ below the right costal margin. Both axillary and femoral pulses were weak. A mid systolic murmur was audible over the upper left sternal edge, the second heart sound was loud in the pulmonary area, and gallop rhythm was present at the cardiac apex. An electrocardiogram showed right atrial enlargement and right ventricular hypertrophy. Chest radiographs indicated pulmonary oedema and moderate cardiomegaly with left atrial enlargement. Cardiac catheterisation 48 hours after birth showed systemic pressures and high oxygen saturations in the pulmonary artery due to persistence of the ductus arteriosus. The mean left atrial pressure was $20 \mathrm{~mm} \mathrm{Hg}$. The injection of contrast medium into the left ventricle showed in the lateral view (Fig. la) two aortic arches with the brachiocephalic trunk, the left carotid and the left subclavian arteries arising from the upper arch. The lower arch, which was of similar size, arose from the aorta at the level of the brachiocephalic trunk and joined the upper arch above an area of coarctation below which the ductus arteriosus arose. It was concluded that this lower arch was a persistent

Requests for reprints to Dr Alberto Cabrera, Alameda Recalde 35B2D, Bilbao, Spain. fifth aortic arch. The frontal view (Fig. 1b) showed a left sided aortic arch and the brachiocephalic trunk as its first branch.

Surgical treatment was performed immediately; the ductus was ligated and the fifth aortic arch was separated from the ascending aorta and used to reconstruct the fourth arch by making an anastomosis of 0.8 $\mathrm{cm}$ diameter between proximal and distal portions of the normal aorta (Fig. 2a and b). Five years later axillary and femoral pulses were normal and no murmurs were audible.

\section{Discussion}

Embryological evidence of a fifth aortic arch became accepted in man in $1922 . .^{8}$ Forty seven years later the necropsy finding of a fifth aortic arch complicated by tricuspid atresia and a divided left atrium was reported. ${ }^{1}$ Anatomically, the fifth aortic arch emerges from the aorta at the proximal portion of the fourth arch and ends in the distal portion of the sixth.

Persistence of a fifth aortic arch has to be differentiated from a retro-oesophageal right subclavian artery and from a double aortic arch. Both can be distinguished by angiography; in the former the frontal view shows the right subclavian and right carotid arteries arising separately from the aorta; in the latter, the lateral view shows superimposition of the double arches in contrast to the separate but parallel positions of the fourth and persistent fifth aortic arches. The differentiations can be confirmed by radiological examination with a barium filled oesophagus, which is compressed laterally or posteriorly by either an anomalous right subclavian artery or by part of a double aortic arch. Further confirmation can be obtained by cross sectional echocardiography.

Persistence of the fifth aortic arch is a different anomaly when associated with pulmonary atresia. It 

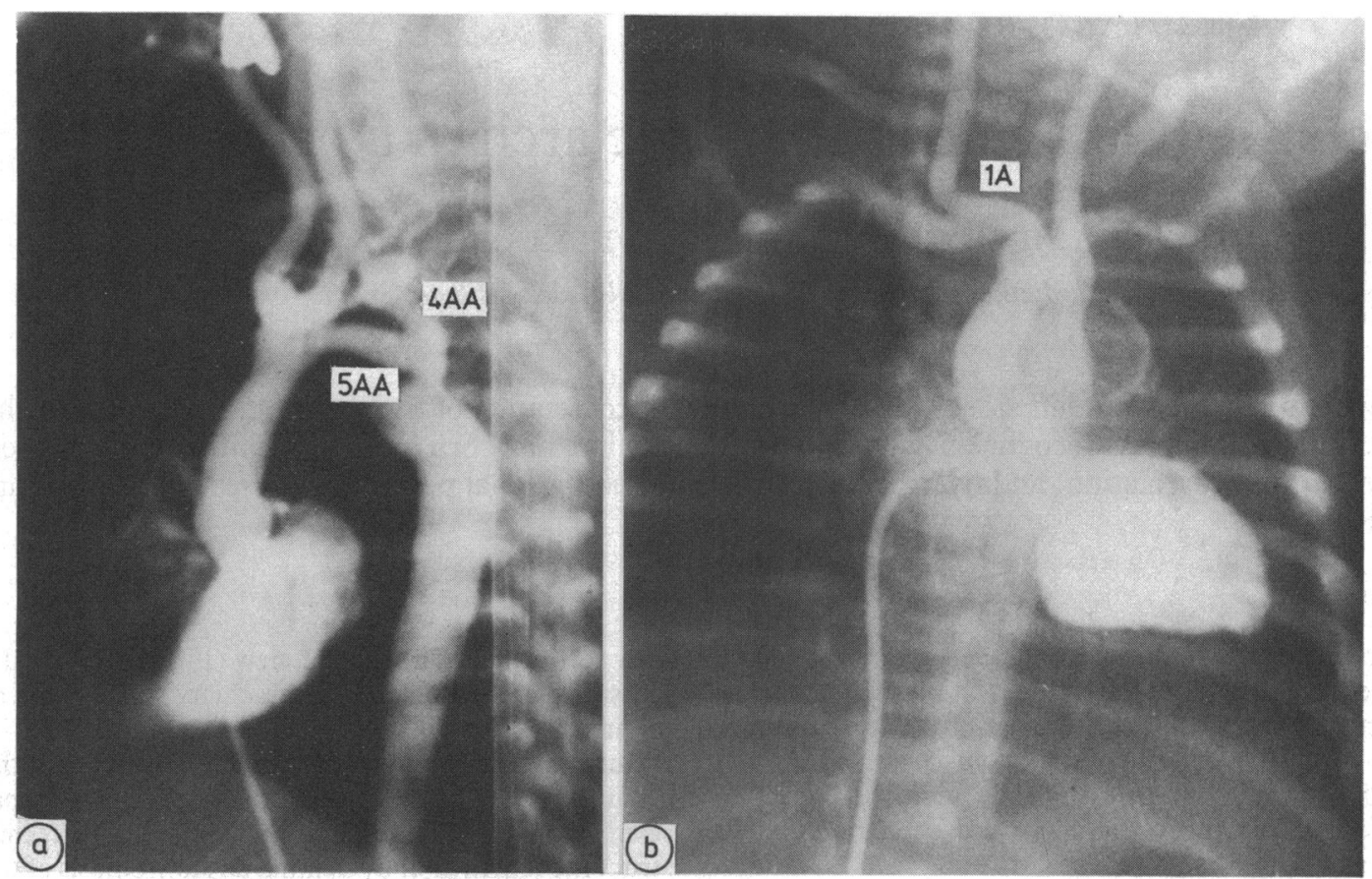

Fig. 1 Angiocardiograms in (a) the lateral and (b) the posteroanterior view after injections of the left ventricle. (a) Two images can be seen, the upper one (4AA) emerging from the supra-aortic trunk, the lower one corresponding to the fifth aortic $\operatorname{arch}(5 A A)$. Coarctation of the aorta and initialfilling of the ductus arteriosus are also seen. (b) The brachiocephalic trunk is well developed and is seen as the first vessel (1A) emerging from the aorta. $4 A A$, fourth aortic arch

can then be a naturally occurring source of pulmonary arterial supply, ${ }^{67}$ is not comparable with the present case report, and would not have been available for incorporation in the surgical repair.
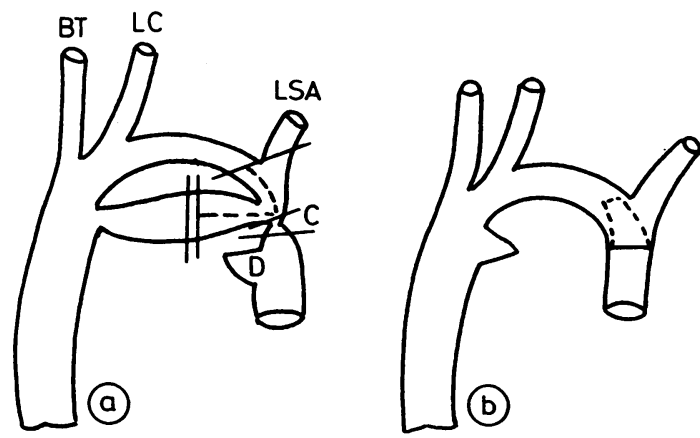

Fig. 2 Diagram showing the position of the fourth (4AA) and fifth (SAA) aortic arches, the persistent ductus $(D)$ and the coarctation $(C)$ before $(a)$ and after $(b)$ surgical repair. $B T$, brachiocephalic trunk; LC, left carotid (artery); LSA, left subclavian artery.

\section{References}

1 van Praagh R, van Praagh S. Persistent fifth arterial arch in man: congenital double lumen aortic arch. $\mathrm{Am} \mathcal{F} \mathrm{Car}$ diol 1969; 24: 279-82.

2 Diaz-Góngora G, Quero Jiménez M, Varón H, Cabrera C, Cabrera R. Persistencia del quinto arco aórtico. Revista Latina Cardiologica 1983; 4: 429-34.

3 Izukawa T, Scott ME, Durrani F, Moes CAF. Persistent left fifth aortic arch in man. Br Heart F 1973; 35: 1190-5.

4 Moes CAF, Izukawa T. Persistent fifth arterial arch; diagnosis during life, with postmortem confirmation. Radiology 1974; 111: 175-6.

5 Lawrence TK, Stiles QR. Persistent fifth aortic arch in man. Am f Dis Child 1975; 129: 1229-31.

6 Macartney FJ, Scott O, Deverall PB. Haemodynamic and anatomical characteristics of pulmonary blood supply in pulmonary atresia with ventricular septal defectincluding a case of persistent fifth aortic arch. Br Heart $\mathcal{f}$ 1974; 36: 1049-60.

7 Macartney FJ, Haworth SG. Investigations of pulmonary atresia with ventricular septal defects. In: Anderson RH, Macartney FG, Shinebourne EA, Tynan M, eds. Paediatric cardiology vol 5. Edinburgh: Churchill Livingstone 1983: 116.

8 Congdon ED. Transformation of the aortic arch system during the development of the human embryo. Contributions to Embryology 1922; 14: 47-110. 